\title{
Biology of Malaria Parasites
}

\author{
John C. Igweh \\ Delta State University, Abraka Nigeria, \\ Nigeria
}

\section{Introduction}

Plasmodium is a genus of parasitic protists. Infection by these organisms in known as malaria. The genus plasmodium was described in 1885 by Ettore Marchiafava and Angelo Celli. Currently over 200 species of this genus are recognized and new species continue to be described.[1]

Of the over 200 known species of plasmodium. At least 11 species infect humans. Other species infect other animals, including monkeys, rodents, and reptiles. The parasite always has two hosts in its life cycle: a mosquito vector and a vertebrate host.[2]

\section{History}

The organism itself was first seen by Lavern on November 6, 1880 at a military hospital in Constantine, Algeria, when he discovered a microgametocyte exflagellating. In 1885, similar organisms were discovered within the blood of birds in Russia. There was brief speculation that birds might be involved in the transmission of malaria; in 1894 Patrick Manson hypothesized that mosquito could transmit malaria. This hypothesis was independently confirmed by the Italian physician Giovanni Battista Grassi working in Italy and the British physician Ronald Ross working in India, both in 1898. [3] Ross demonstrated the existence of Plasmodium in the wall of the midgut and salivary glands of a Culex mosquito using bird species as the vertebrate host. For this discovery he won the Noble Prize in 1902. Grassi showed that human malaria could only be transmitted by Anopheles mosquito. It is worth noting, however, that for some species the vector may not be mosquito. [ $\left.{ }^{4}\right]$

\section{Biology}

The genome of four plasmodium species - plasmodium falciparum, Plasmodium knowlest, Plasmodium vivax and Plasmodium yoelii - have been sequenced. All these species have genomes of about 25 megabase organized into 14 chromosomes consistent with earlier estimates. The chromosomes vary in length from 500 kilobases to 3.5 megabases and it is presumed that this is the pattern throughout the genus. [5]

\section{Diagnostic characteristics of the genus Plasmodium}

- Merogony occur both in erythrocytes and other tissues 
- Merozites, schizonts or gametocytes can be seen within erythrocytes and may displace the host nucleus

- Merzoites have a "signet-ring" appearance due to a large vacuole that forces the parasite's nucleus to one pole

- Schizonts are round to oval inclusions that contain the deeply staining merozoites

- Forms gamonts in erythrocytes

- Gametocytes are "halter-shaped" similar Haemoproteus but the pigment granules are more confined

- Hemozoin is present

- Vectors are either mosquito's or sand flies

- Vertebrate hosts include mammals, bird and reptiles

\section{Life cycle}

The life cycle of Plasmodium while complex is similar to several other species in the Haemosporidia.

All the Plasmodium species causing malaria in humans are transmitted by mosquito species of the genus Anopheles. Species of the mosquito genera Aedes, Culex, Mansonia and Theobaldia can also transmitted malaria but not to humans. Bird malaria is commonly carried by species belonging to the genus Culex. The life cycle of Plasmodium was discovered by Ross who worked with species from the genus Culex.[ [6]

Both sexes of mosquito live on nectar. Because nectar's protein content alone is insufficient for oogenesis (egg production) one or more blood meals is needed by the female. Only female mosquito bite.

Sporozoites from the saliva of a biting female mosquito are transmitted to either the blood or the lymphatic system of the recipient. It has been known for some time now that parasites block the salivary ducts of the mosquito and as a consequence the insect normally requires multiple attempts to obtain blood. The reason for this has not been clear. It is not known that the multiple attempts by the mosquito may contribute to immunological tolerance of the parasite. [4] The majority of sporozoites appear to be injected into the subcutaneous tissue from which they migrate into the capillaries. A proportion is ingested by macrophages and still others are taken up by the lymphatic system where they are presumably destroyed. $10 \%$ of the parasites inoculated by the mosquitoes may remain in the skin where they may develop into infective merozoites. [5,7,8]

\section{Hepatic stages}

The majority of sporozoites migrate to the liver and invade hepatocytes. For reasons that is currently unclear sporozoites typically penetrate several hepatocytes before choosing one to reside within. $\left[{ }^{9}\right]$ The sporozoite then matures in the hepatocyte to a schizont containing many merozoites in it. In some Plasmodium species, such as Plasmodium vivax and Plasmoduim ovale, the parasite in the hepatocyte may not achieve maturation to a schizont immediately but remain as a latent or dormant form and called a hypnozoite. Although Plasmodium falciparum is not considered to have a hypnozoite form. This may not be entirely correct. This stage may be as short as 48 hours in the rodent parasite and as long as 15 days in P. malaria in humans. $[10,11]$ 
There is considerable variation in the appearance of the blood between individuals experimentally inoculated at the same time. Even within a single experimental individual there may be considerable variation in the maturity of the hepatic forms seen on liver biopsy.[12]

A proportion of the hepatic stages may remain within the liver for considerable time - a form known as hypnozoites. Reactivation of the hynozoites have been reported for up to 30 years after the initial infection in humans. The factors precipitating this reactivation are not known. In the species Plasmodium ovale and Plasmodium vivax. It is not yet known if hypnozoite reactivation occurs with any of the remaining species that infect humans but this is presumed to be the case.[13,14]

The development from the hepatic stages to the erythrocyte stages have, until very recently, been obscure. In 2006 it was showed that the parasite buds off the hepatocytes in merosomes containing hundreds of thousand of merozoties. These merosomes lodge in the pulmonary capillaries and slowly disintegrate there over 48 - 72 hours releasing merozoites. Erythrocyte invasion is enhanced when blood flow is slow and the cells tightly packed: both of these conditions are found in the alveolar capillaries.[15,16]

\section{Erythrocyte stage}

After entering the erythrocyte, the merozoite lose one of their members, the apical rings, conoid and the rhopteries. Phagotropy commences and both smooth and granular endoplasmic reticulum because prominent. The nucleus may become lobulated.[16]

Within the erythrocytes the merozoite grow first to a ring-shaped form and then to a larger trophozoite form. In the schizont stage, the parasite divides several times to produce new merozoites. Which leave the red blood cells and travel within the bloodstream to invade new red blood cells. The parasite feeds by ingesting hemoglobin and other materials from red blood cells and serum. The feeding process damages the erythrocytes. Details of process have not been studied in species other than Plasmodium falciparum. so generalization may be premature at this time.

Erythrocytes infected by Plasmodium falciparum tend to form clumps - rosettes - and these have been linked to pathology caused by vascular occlusion. This rosette formation may be inhibited by heparin. This agent has been used in the past as part of the treatment of malaria but was abandoned because of an increased risk of haemorrhage. Low molecular weight heparin also disrupts rosette formation and may have a lower risk of bleeding in malaria.[17]

\section{Merozoites}

The budding of the merozoites from interconnected cytoplasmic masses (pseudocytomeres) is a complex process. At the tip of each bud a thickened region of pellicle gives rise to the apical rings and conoid. As development proceeds an aggregation of smooth membranes and the nucleus enter the base of the bud. The cytoplasm contains numerous large ribosomes. synchronous multiple cytoplasmic cleavage of the mature schizont results in the formation of numerous uninucleate merozoites.

Escape of the merozoites from the erythrocyte has also been studies. The erythrocyte swells under osmotic pressure. A pore opens in the erythrocyte membrane and 1-2 meorozites 
escape. This is followed by an eversion of the entire erythrocyte membrane. An action that propels the merozoites into the blood stream.

Invasion of erythrocyte precursors has only recently been studied. The earliest stage susceptible to infection were the erythroblasts - the stage immediately preceding the reticulocyte stage which in turn is the immediate precursor to the mature erythrocyte. Invasion of the erythrocyte is inhibited by angiotensin 2 . This is normally metabolized by erythrocytes to angiotensin (Ang) IV and Ang - (1-7). Parasite infection decreased the Ang (1-7) levels and completely abolished Ang IV formation. Ang - (1-7), like its parent molecule, is capable of decreasing the level of infection. The mechanism of inhibition seems likely to be an inhibition of protein Kinase A activity within the erythrocyte.

\section{Placental malaria}

More than a hundred late-stage trophozoites or early schizont infected erythrocytes of $P$. falciparum in a case of placental malaria of a Tanzanian woman were found to form a nidus in an intervillous space of placenta. While such a concentration of parasite in placental malaria is rare, placental malaria cannot give rise to persistent infection as pregnancy in humans normally lasts only 9 months. We have also found this kind of placental infection in our own studies.[18]

\section{Gametocytes}

Most merozoites continue this explicative cycle but some merozoites differentiate into male or female sexual forms (gametocytes) (also in the blood), which are taken up by the female mosquito. This process of differentiation into gametocytes occur in the bone marrow. Five distinct morphological stages have recognized (stage I-V). Female gametocytes are produced about four times as commonly as male. In chonic infections in humans the gametocytes are often the only forms found in the blood. Incidentally the characteristic form of the female gametocytes in Plasmodium falciparum gave rise to this specie's name.

Gametocytes appear in the blood after a number of days post infection. In P. falciprum infections they appear after 7 to 15 days while on others they appear after 1 to 3 days. The ratio of asexual to sexual forms is between 10:1 and 156:1. The half life of the gametocytes has been estimated to be between 2 and 3 days but some are known to persist for up to four weeks.[18,19]

The five recognized morphological stages were first described by Field and Shute in 1956.

One constant feature of the gametocytes in all stages that distinguishes them from the asexual forms is the presence of a pellicular complex. This originates in small membranous vesicle observed beneath the gametocytes plasmalemma in late stage I. The structure itself consists of a subpellicular membrane vacuole. Deep to this is an array of longitudinally oriented microtubules. This structure is likely to be relatively inflexible and may help to explain the lack of amoeboid forms observed in asexual parasites.

Early stage one gametocytes are very difficult to distinguish from small round trophozoites. Later stages can be distinguished by the distribution of pigment granulues. Under the electron microscope the formation of the subpellicular membrane and a smooth plasma 
membrane are recognizable. The nuclei are recognizably dimorphic into male and female. These forms may be found between 0 and 2 in $P$ falciprum infections.

In stage two gametocytes becomes D shaped. The nucleus may occupy a terminal end of the cell or lie along its length. Early spindle formation may be visible. These forms are found between days 1 to day 4 in $P$ falciprum infections.

In stage three the erythrocyte becomes distorted. A staining difference between the male and female gametocytes is apparent (male stain pink while female stain faint blue with the usual stains). The male nucleus is noticeably larger than the female and more lobulated. The female cytoplasm has more ribosomes, endoplasmic reticulum and mitochondria.

In stage four erythrocytes is clearly deformed and the gametocyte is elongated. The male gametocytes stain red while the female stain violet blue. In the male pigment granules are scattered while in the female they are denser. In the male the kinetochores of each chromosomes are located over a nuclear pore. Osmophilic bodies are found in both but are more numerous in the female. These forms are found between day 6 and day 10 in $P$ falciparum infections.

In stage five the gametocytes are clearly recognizable on light microscopy with the typical banana shaped female gametocytes. The subpellicular microtubules depolymerise but the membrane itself remains. The male gametocyte exhibit a dramatic reduction in ribosomal density. Very few mitochondria are retained and the nucleus enlarges with a kinetochore complex attached to the nuclear envelope. In the female gametocytes there are numerous mitochondria, ribosome's and osmophillic bodies. The nucleus is small with a transcription factory.

Stages other than five are not normally found in the peripheral blood. For reasons not yet understood stage 1 to IV are sequestered preferentially in the bone marrow and spleen. Stage $\mathrm{V}$ gametocytes only become infections to mosquito's after a further two days of circulation.

\section{Infection of mosquito}

In the mosquito's midgut, the gametocytes develop into gametes and fertilize each other, forming motile zygotes called ookinetes. It has been shown that up to $50 \%$ of the ookinetes may undergo apoptosis within the mighut. The reason for this behavior is unknown. While in the mosquito gut the parasites form thin cytoplasmic extensions to communicate with each other. These structures persist from the time of gametocyte activation until the zygote transforms into an ookinete. The function of these tubular structures remains to be discovered.[20]

The ookinetes penetrate and escape the midgut, then embed themselves onto the exterior of the gut membrane. As in the liver the parasite tends to invade a number of cells before choosing one to reside in. the reason for the behavior is not known. Here they divide many times to produce large number of tiny elongated sporozoites. These sporozoites migrate to the salivary glands of the mosquito where they are injected into the blood and subcutaneous tissue of the next host the mosquito bites. 
The escape of the gametocytes from the erythrocytes has been until recently obscure. The parasitophorous vacuole membrane ruptures at multiple sites within less than a minute following ingestion. This process may be inhibited by cysteine protease inhibitors. After this rupture of the vacuole the subpellicular membrane begins to disintegrate. This process also can be inhibited by aspartic and the cysteine/ serine protease inhibitors. Approximately 15 minutes post-activation. The erythrocytes membrane rupture at a single breaking point a third process that can be interrupted by protease inhibitors.

Infection of the mosquito has noticeable effects on the host. The presence of the parasite induces apoptosis of the egg follicles.

\section{Discussion}

The pattern alternation of sexual and asexual reproduction which may seem confusing at first is a very common pattern in parasite species. The evolutionary advantages of this type of life cycle were recognized by Gregor Mendel.

Under favorable conditions asexual reproduction is superior to sexual as the parent is well adapted to its environment and its descendents share these genes. Transferring to a new host or in times of stress, sexual reproduction is generally superior as this produces a shuffling of genes which on average at a population level will produce individuals better adapted to the new environment.

Given that this parasite spends part of its life cycle in two different hosts it must use a proportion of its available resources within each host. The proportion utilized is currently unknown. Empirical estimates of this parameter are desirable for modeling of its life cycle.

\section{Dormant Form}

\section{Plasmodium falciparum malaria}

A report of $P$. falciparum malaria in a patient with sickle cell anemia four years after exposure to the parasite has been published. A second report that $P$. falciparum malaria had become symptomatic eight years after leaving an endemic area has also been published.[21]

A third case of an apparent recurrence nine years after leaving an endemic area of $P$. falciparum malaria has now been reported. A fourth case of recurrence in a patient with lung cancer has been reported. Two cases in pregnant woman both from Africa but who had not lived there for over a year have been reported.

A case of congenital malaria due to both $P$. falciparum and $P$. malariae has been reported in a child born to a woman from Ghana, a malaria endemic area, despite the mother having emigrated to Austria eighteen months before and never having returned. A second case of congenital malaria in twins due to $P$. falciparum has been reported. The mother had left Togo 14 months before the diagnosis, had not returned in the interim and was never diagnosed with malaria during pregnancy.[24,25]

It seems that at least occasionally P. falciparum has a dormant stage. If this is in fact the case, eradication or control of this organism may be more difficult than previously believed.[25,26] 


\section{Drug induced}

Developmental arrest was induced by in vitro culture of $P$. falciparum in the presence of sub lethal concentration of artemisinin. The drug induced a subpopulation of ring stage into developmental arrest. At the molecular level this is associated with over- expression of heat shock and erythrocyte binding surface protein with the reduced expression of a cell-cycle regular and a DNA biosynthesis protein.

The schizont stage-infected erythrocyte in an experimental culture of P. falciparum, F32 was suppressed to a low level with the use of atovaquone. The parasite resumed growth several days after the drug was removed from the culture.[28]

\section{Biological refuges}

Macrophages containing merozoites dispersed on their cytoplasm. Called 'merophores', were observed in $P$. vinkei petteri- an organism that causes murine malaria. Similar merophores were found in the polymorph leukocytes and macrophages of other murine malaria parasite, $P$. yoelii nigeriensis and $P$. chabudi chaaudi. All these species unlike $P$. falciparum are known to produce hyponozoites that may cause a relapse. The finding of Landau et al. on the presence of malaria parasites inside lymphatics suggest a mechanism for the recrudescence and chronicity of malaria infections. [29,30,]

\section{Evolution}

As of 2007, DNA sequences are available from less than sixty species of Plasmodium and most of these are from species infecting either rodent or primate hosts. The evolutionary outline given here should be regarded as speculative, and subject to revision as more data becomes available.[31,32,]

The 'Apicomplexa (the phylum to which Plasmodium belong) are thought to have originated within the Dinofagellates -a large group of photosynthetic protists. It is thought that the ancestors of the Apicomplexa were originally prey organisms that evolved the ability to invade the intestinal cells and subsequently lost their photosynthetic ability. Many of the species within the Apicomplexa still possess plastids (the organelle in which photosynthesis occur in photosynthetic eukaryotes), and some that lack plastids nonetheless have evidence of plastid genes within their genomes. In the majority of such species, the plastids are not capable of photosynthesis. Their function is not known, but there is suggestive evidence that they may be involved in reproduction.[33]

Some extant dinoflagellates, however, can invade the bodies of jellyfish and continue to photosynthesise, which is possible because jellyfish bodies are almost transparent. In host organisms with opaque bodies, such an ability would most likely rapidly be lost. The 2008 description of a photosynthetic protist related to the Apicomplexa with a functional plastid supports this hypothesis.[32]

Current (2007) theory suggests that the genera Plasmodium Hepatocystis and Haemoprotus evolved from one or more Leucoytozoon species. Parasites of the genus Leucocytozoon infect white blood cells (Leukocytes) and liver and spleen cells, and are transmitted by 'black flies' (Simulium species) ----- a large genus related to the mosquitoes. 
It is thought that Leucocytozoon evolved from a parasite that spread through the orofaecal route and which infected intestinal wall. At some point parasites evolved the ability to infect the liver. This pattern is seen in the genus Cryptosporidium, to which Plasmodium is distantly related. At some later point this ancestor developed the ability to infect blood cell and to survive and infect mosquitoes. Once vector transmission was firmly established, the previous orofecal route of transmission was lost.

Molecular evidence suggests that a reptile - specifically a squamte - was the first vertebrate host of Plasmodium. Birds were the second vertebrate hosts with mammals being the most recent group of vertebrates infected.

Leukocytes, hepatocytes and most spleen cells actively phagocytes particulate matter, which makes the parasite's entry into the cell easier. The mechanism of entry of Plasmodium species into erythrocytes is still very unclear, as it takes place in less than 30 seconds. It is not yet known if this mechanism evolved before mosquitoes became the main vectors for transmission of Plasmodium.[35]

The genus Plasmodium evolved (presumably from its Leucocytozoon ancestor) about 130 million years ago, a period that is coincidental with the rapid spread of the angiosperms (flowering plants). This expansion in the angiosperms is thought to be due to at least one gene duplication event. It seems probable that the increase in the number of flowers led to an increase in the number of mosquitoes and their contact with vertebrates.[36]

Mosquitoes evolved in what is now South America about 230 million years ago. There are over 3500 species recognized, but to date their evolution has not been well worked out, so a number of gaps in our knowledge of the evolution of Plasmodium remain. There is evidence of a recent expansion of Anopheles gambiae and Anopheles arabiensis populations in the late Pleistocene in Nigeria.[37]

The reason why a relatively limited number of mosquitoes should be such successful vectors of multiple diseases is not yet known. It has been shown that, among the most common disease - spreading mosquitoes, the symbiont bacterium Wolbachia are not normally present. It has been shown that infection with Wolbachia can reduce the ability of some viruses and Plasmodium to infect the mosquito, and that this effect is Wolbachia-strain specific.

\section{Classification}

\section{Taxonomy}

Plasmodium belong to the family plasmodium, order Haemosporidia and phylum Apicomplexa. There are currently 450 recognized species in this order. Many species of this order are undergoing reexamination of their taxonomy with DNA analysis. It seems likely that many of these species will be re-assigned after these studies have been completed. For this reason the entire order is outlined here.[38,39]

Order Haemoporida

Family Haemoproteidae

- Genus Haemoproteus

- Subgenus Parahaemoproteus 
- Subgenus Haemoproteus

Family Garniidae

- Genus Fallisia Lainson, Landau \& Shaw 1974

- Subgenus Fallisia

- Subgenus Plasmodioides

- Genus Garnia

- Genus Progarnia

Family Leucocytozoidae

- Genus Leucocytozoon

- Subgenus Leucocytozoon

- Subgenus Akiba

Family Plasmodiidae

- Genus Bioccala

- Genus Billbraya

- Genus Dionisiu

- Genus Hepatocystis

- Genus Mesnilium

- Genus Nycteria

- Genus Plasmodium

- $\quad$ Subgenus Asiamoeba Telford 1988

- $\quad$ Subgenus Bennettinia Valkiunas 1997

- $\quad$ Subgenus Carinamoeba Garnham 1996

- $\quad$ Subgenus Giovannolaia Corradetti, Garnham \& Laird 1963

- $\quad$ Subgenus Haemamoebe Grassi \& Feletti 1890

- $\quad$ Subgenus Huffia Garnham \& Laird 1963

- $\quad$ Subgenus Lucertaemoba Telford 1988

- $\quad$ Subgenus Laverania Bray 1963

- $\quad$ Subgenus Novyella Corradtti, Garnham \& Laird 1963

- $\quad$ Subgenus Ophidiella Garnham 1966

- $\quad$ Subgenus Plasmodium Bray 1963 emend, Garnham 1964

- $\quad$ Subgenus paraplasmodium Telford 1988

- Subgenus Sauramoeba Garnham 1966

- $\quad$ Subgenus Vinekeia Garnham 1964

- Genus Polychromophilus

- Genus Ravella

- Genus Saurcytozoon

The eleven 'Asian' species included here form a clade with $P$. vivax being clearly closely related as are $P$. knowsell and $P$. coatneyi; similarly $P$. brazilium and $P$. malaria are related. $P$. hylobati and $P$. inui are closely related. $P$. gonderi appear to be more closely related to $P$. vivax than P. malaria.

P. coatneyi and $P$. inui appear to be closely related to $P$. vivax.[38] 
P. ovale is more closely related to $P$. malaria than to $P$. vivax.

Within the 'Asian' clade are three unnamed potential species. One infect each of the two chimpanzee subspecies included in the study (Pan troglodytes troglodytes and pan troglodytes schweinfurthii). These appear to be related to the $P$. vivax $P$. simium clade.

Two unnamed potential species infect the bonbo (Pan paniscus) and these are related to the P. malaria P. brazillium calde.

\section{Subgenera}

The full taxonomic name of a species includes the subgenus but is often omitted. The full name indicates some features of the morphology and type of host species. Sixteen subgenera are currently recognized.

The avian species were discovered soon after the description of $P$ falciparum and a variety of generic names were created. These were subsequently placed into the genus Plasmodium although some workers continued to use the genera Laverinia and Proteosoma for $P$. falciparum and the avian species respectively. The $5^{\text {th }}$ and $6^{\text {th }}$ Congresses of Malaria held at Istanbul (1953) and Lisbon (1958) recommended the creation and use of subgenera in this genus. Laverinia was applied to the species infecting human and Haemamoeba to those infecting lizard and birds. This proposal was not universally accepted. Bray in 1955 proposed a definition for the subgenus Plasmodium and a second for the subgenus Laverinia in 1958. Garnham described a third subgenus - Vinckeia - in 1964.[40]

\section{Mammal infecting species}

Two species in the subgenus Laverania are curreIntly recognized: $P$. falciparum and $P$. reichenowi. Three additional species - Plasmodium billbrayi, Plasmodium billeollnisi and Plasmodium gaboni - may also exist (based on molecular data) but a full description of these species have not yet been published. The presence of elongated gametocytes in several of the avian subgenus and in Laverania in addition to a number of clinical features suggested that these might be closely related. This is no longer thought to be the case.[11]

The type species is Plasmodium falciparum.

Species infecting monkeys and apes (the higher primate other than those in the subgenus Laverania are placed in the subgenus Plasmodium. The position of the recently described Plasmodium Gor A and Plasmodium Gor $B$ has not yet been settled. The distinction between $P$. falciparum and $P$. reichenowi and the other species infection higher primates was based on the morphological findings but have since been confirmed by DNA analysis.[42,43]

The type speces is Plasmodium malaria

Parasites infecting other mammals including lower primates (lemurs and others) are classified in the subgenus Vinckeia. Vinckeia while previously considered to be something of a taxonomic 'rag has been recently shown - perhaps rather surprisingly - to form a coherent grouping.

The type species is Plasmodium bubalis.[44,45,46,47,48] 


\section{Bird infecting species}

The remaining groupings are based on the morphology of the parasites. Revision to this system are likely to occur in the future as more species are subject to analysis of their DNA.

The four subgenera Giovannolaia, Haemamoeba, Huffia and Novyella were created by Corradetti et al. for the known avian malaria species. A fifth - Bannettinia - was created in 1997 by Valkiunas. The relationship between the subgenera are the matter of current investigation. Marinsen et al. 's recent (2006) paper outlines what is currently (2007) known. The subgenera Haemamoeba, Huffia, and Bennettinia appear to be monophyletic. Novyella appear to be well defined with occasional exceptions. The subgenus Giovannolaia need revision. $[49,50]$

P. juxtanucleare is currently (2007) the only known member of the subgenus Bennettinia.

Nyssorhynchus is an extinct subgenus of Plasmodium. It has one known member - Plasmodium dominium[51].

\section{Reptile infecting species}

Unlike the mammalian and bird malaria those species (more than 90 currently known) that infect reptiles have been more difficult to classify.

In 1966 Garnham classified those with large schizonts as Sauramoeba, those with small schizont as Carinamoeba and the single then known species infecting snakes (Plasmodium wenyoni) as Ophidiella. He was aware of the arbitrariness of this system and that it might not prove to be biologically valid. This scheme was used as the basis for the currently accepted system.[52]

These species have since been divided in to 8 genera -Asiamoeba, Carinamoeba, Fallisia Garnia, Lacertamoeba, and Paraplasmodium and Sauramoeba. Three of these genera (Asiamoeba, Lacertamoeba and Plaraplasmodium) were created by Telford in 1988. Another species (Billbraya australis) described in 1990 by Paperna and landau and is the only known species in this genus. This species may turn out to be another subgenus of lizard infecting Plasmodium.

With the exception of P. elongatum the exoerythrocytic stage occur in the endothelial cells and those of the macrophage - lymphoid system. The exoerthrocytic stage of P. elongatum parasite the blood forming celss.

The various subgenera are first distinguished on the basis of the morphology of the mature gametocytes. Those of subgenus Haemamoeba are round oval while those of the subgenera Giovannolaia, Hiffia and Novyella are elongated. These latter genera are distinguished on the basis of the size of the schizont: Giovannolaia and Huffia have large schizonts while those of Novyella are small.[52]

Species in the subgenus Bennettinia have the following characteristics:

The type species is Plasmodium juxtanucleare.

Species in the subgenus Giovannolaia have the following characteristics: 
- Schizonts conyain plentiful cytoplasm, are larger than the host cell nucleus and frequently displace it. They are found only in mature erythrocytes.

- Gametocytes are elongated

- Exoerythrocytic schizony occur in the mononuclear phagocyte system.

The type species is Plasmodium circumflexum.

Species in the subgenus Haeamoeba have the following characteristics:

- Mature schizonts are larger than the host cell nucleus and commonly distplace it.

- Gametocytes are large, round, oval or irregular in shape and are substantially larger than the host nucleus.

The type species is Plasmodium relictum

Species in the subgenus Huffia have the following characteristics:

- Mature schizonts, while varying in shape and size, contain plentiful cytoplasm and are commonly found in immature erythrocytes.

- Gametocytes are elongated.

The type species is Plasmodium elongated

The type of spices subgenus Novyella have the following characteristics:

- Mature schizonts are either smaller than or only slightly larger than the host nucleus they contain scanty cytoplasm.

- Gametocytes are elongated. Sexual stage in this subgenus resemble those of Haemoproteus.

- Exoerythrocytic schizongony occur in the mononuclear phagocyte system[52]

The type species is Plasmodium vaughni

\section{Malaria parasites}

Malaria parasites are micro-organisms that belong to the genus Plasmodium. There are more than 100 species of Plasmodium, which can infect many animal species such as reptiles birds, and various mammals. Four species of Plasmodium have long been recognized to infect humans in nature. In addition there is one species that naturally infect macaques which has recently been recognized to be a cause of zoonotic malaria in humans. (There are some additional species which can, exceptionally or under experimental conditions, infect humans.)

- $\quad$ P. falciparum, which is found worldwide in tropical and subtropical. It is estimated that every year approximately I million people are killed by $P$. falciparum, especially in Africa where this species predominates. P. falciparum, can cause severe malaria because it multiples rapidly in the blood, and can thus cause severe blood loss (anemia). In addition, the infected parasites can clog small blood vessels. When this occur in the brain, cerebral malaria results, a complication that can be fatal.

- $\quad P$. vivax, which is found mostly in Asia, Latin America, and in some parts of Africa. Because of the population densities especially in Asia it is probably the most prevalent human malaria parasite. P. vivax (as well as $P$. ovale), has dormant liver stages ("hypnozoites") that can activate and invade the blood ("relapse") several months or years after the infecting mosquito bite. 
- $\quad \boldsymbol{P}$. ovale is found mostly in Africa (especially West Africa) and the island of the western pacific. It is biologically and morphologically very similar of $P$ vivax. however, differently from $P$. vivax, it can infect individuals who are negative for the Duffy blood group, which is the case for many residents of sub-Saharan Africa. This explains the greater prevalence of $P$. ovale (rather than $P$. vivax) in most of Africa.

- $\quad \boldsymbol{P}$. malaria, found worldwide, is the only human malaria parasite species that has a quartan cycle (three-day cycle). (The three other species have a tertian, two-day cycle.) If untreated, $P$. malaria cause a long-lasting, chronic infection that in some cases can last a lifetime. In some chronically infected patients $P$. malaria can cause serious complications such as the nephritic syndrome.

- $\quad$ P. knowlesi is found throughout Southern Asia as a natural pathogen of long-tailed and pigtailed macaques. It has recently been shown to be a significant cause of zoonotic malaria in that region, particularly in Malaysia. P. knowlesi has a 24 -hour replication cycle and so can rapidly progress from an uncomplicated to a severe infection.

\section{Cellular and molecular biology of Plasmodium}

Members of the genus Plasmodium are eukaryotic microbes. Therefore, the cell and molecular biology of Plasmodium will be similar to other eukaryotes. A unique feature of the malaria parasite is its intracellular lifestyle. Because of its intracellular location the parasite has an intimate relationship with its host cell which can be described at the cellular and molecular levels. In particular, the parasite must enter the host cell, and once inside, it modifies the host cell. The molecular and cellular biology of host-parasite interactions involved in these two processes will be discussed.

\section{Host cell invasion}

Malaria parasites are members of the Apicomplexa. Apicomplexa are characterized by a set of organelles found in some stage of the parasite's life cycle. These organelles, collectively known as apical organelles because of their localization at one end of the parasite, are involved in interactions between the parasite and host. In particular, the apical organelles have been implicated in the process of host celll invasion. In the case of Plasmodium, three distinct invasive forms have been identified: sporozoite, merozoite, and ookinete (see Plasmodium Life Cycle). The following discussion focuses on the cellular biology of merozoites and erythrocyte invasion. Reference to other Apicomplexa and Plasmodium sporozoites will be made to illustrate common features.

Merozoites rapidly (approximately 20sec.) and specifically enter erythrocytes. This specificity is manifested both for erythrocytes as the preferred host cell type and for a particular host species, thus implying receptor - ligand interactions. Erythrocyte invasion is a complicated process which is only partially understood at the molecular and cellular levels.[53] Four distinct steps in the invasion process can be recognized:

1. Initial merozoites binding

2. Reorientation and erythrocyte deformation

3. Junction formation

4. Parasite entry 


\section{Merozoite surface proteins and host- parasite interaction}

The initial interaction between the merozoite and the erythrocyte is probably a random collision and presumably involves reversible interactions between proteins on the merozoite surface and the host erythrocyte. Several merozoite surface proteins have been described. The best characterized is merozoite surface protein - 1 (MSP-1). Circumstantial evidence implicating MSP - I in erythrocyte invasion include its uniform distribution over the merozoite surface and the observation that antibodies against MSP-I inhibit invasion. [ $\left.{ }^{54}\right]$ In addition, MSP-I does bind to band 3.[55] However, a role for MSP-I in invasion has not been definitively demonstrated. Similarly, the circumsporozite protein (CSP) probably plays a role in targeting sporozoites to hepatocytes by interacting with heparin sulfate proteoglycans . [56]

Another interacting aspect of MSP-I is the proteolytic processing that is coincident with merozoite maturation and invasion.[57] A primary processing occurs at the time of merozite maturation and result in the formation of several polypeptides held together in a noncovalent complex. A secondary processing occurs coincident with merozoite invasion at a site near the C-terminus. The non-covalent complex of MSP-I polypeptide fragments is shed from the merozoite surface following proteolysis and only a small C-terminal fragment is carried into the erythrocyte. This loss of the MSP-I complex may correlate with the loss of the 'fuzzy' coat during merozoite invasion. The C-terminal fragment is attached to the merozoite surface by a GPI anchor and consists of two EGF-like modules. EGF-like modules are found in a variety of protein and are usually implicated in protein-protein interactions. One possibility is that the secondary proteolytic processing functions to expose the EGF-like modules which strengthen the interactions between merozoite and erythrocyte. The importance of MSP-I and its processing are implied from the following observations:

- Vaccination with the EGF-like modules can protect against malaria, and

- Inhibition of the proeolytic processing blocks merozoite invasion.

The exact role(s) which MSP-I and its processing play in the merozoite invasion process are not known.

\section{Reorientation and secretory organelles}

After binding to erythrocyte, the parasite reorient itself so that the 'apical end' of the parasite is juxtaposed to the erythrocyte membrane. This merozoite reorientation also coincides with a transient erythrocyte deformation. Apical membrane antigen-I (AMA-I) has been implicated in this reorientation.[58] AMA-I is a transmembrane protein localized at the apical end of the merozoite and bind erythrocytes. Antibodies against AMA-I do not interfer with the initial contact between merozoite and erythrocytes thus suggesting that AMA-I is not involved in merozoite attachment. But antibodies against AMA-I prevent the reorientation of the merozoite and thereby block merozoite invasion.

specialized secretory organelles are located at the invasive stages of apicomplexan parasites. Three morphologically distinct apical organelles are detected by electron microscopy; mocronemes, rhoptries, and dense granules. Dense granules are not always included with the apical oganelles and probably represent a heterogeneous population of secretory vesicles. 
The contents of the apical organelles are expelled as the parasite invades, thus suggesting that these organelles play some role in invasion. Experiments in Toxoplasma gondii indicate that the micronemes are expelled first and occur with initial contact between the parasite and host.[59] An increase in the cytoplasmic concentration of calcium is associated with microneme discharge. [60] as is typical of regulated secretion in other eukaryotes.

Dense granule contents are released after the parasite has completed its entry, and therefore, are usually implicated in the modification of the host cell. For RESA is localized to dense granules in merozoites and is transported to the host erythrocyte membrane shortly after merozoite invasion.[61] However, subtilisin-like proteases, which are implicated in the secondary proteolytic processing of MSP-I (discussed above), have also been localized to Plasmodium dense granules.[62,63] If MSP-I processing is catalyzed by these proteases, then at least some dense granules must be discharged at the time of invasion.

\section{Specific interactions and junction formation}

Following merozoite reorientation and microneme discharge a junction forms between the parasite and host cell. Presumably, microneme proteins are important for junction formation. Proteins localized to the micromenes include:

- $\quad$ EBA-175, a $175 \mathrm{kDa}$ 'erythrocyte binding antigen P. falciparum

- $\quad$ DBP, Duffy-binding protein from P. vivax and P. knowlesi

- SSP2, Plasmodium sporozoite surface protein-2. Also known as TRAP (thrombospondinrelated adhesive protein).

- Proteins with homology to SSP2/TRAP from Toxoplasma (MIC2), Eimeria (Etp100), and Cryptosporidium

- CTRP, circumsporozoite- and TRAP- related protein of Plasmodium found in the ookinete stage.

Of particular note are EBA-175 and DBP which recognize sialic acid residues of the glycohorins and the Duffy antigen, respectively. In other words, these parasite protein are probably involved in receptor-ligand interaction with proteins exposed on the erythrocyte surface. Disruption of the EBA-175 gene results in the parasite switchingfrom a sialic aciddependentpathway to a sialic acid-independent pathway [ $\left.{ }^{64}\right]$, indicating that there is some redundancy with regards to the receptor - ligand interactions.

Comparison of sequences of EBA-175 and DBP reveal conserved structural features. These include trans-membrane domains and receptor-binding domains.[65] The receptor-binding activity has been mapped to a domain in which the cysteine and aromatic amino acid residues are conserved between species. This putative binding domain is duplicated in EBA175. The topography of the trans-membrane domain is consistent with the parasite ligands being integral membrane proteins with the receptor-binding domain exposed on the merozoite surface following microneme discharge.

The other microneme protein in the 'TRAP' family have also been implicated in locomotion and / or cell invasion. [66] All of these proteins have domains that are presumably involved in cell-cell adhesion, as N-terminal single sequences and trans-membrane domains at their C- terminal. 


\section{In summary}

- An electron-dense junction forms between the apical end of the merozoite and host erythrocyte membrane immediately after reorientation.

- Tight junction formation and microneme release occur at about the same time

- Proteins localized to the micronemes bind to receptors on the erythrocyte surface

These observations suggest that the junction represents a strong connection between the erythrocyte and the merozoite which is mediated by receptor-ligand interaction. Junction formation may be initiated by microneme discharge which exposes the receptor-binding domains of parasite ligands. This mechanism for initiating a tight host-parasite interaction is probably similar in other invasive stages of apicomplexan parasites.

\section{Parasite entry}

Apicomplexan parasite actively invade host cells and entry is not due to uptake or phagocytosis by the host cell. This is particularly evident in the case of the erythrocyte which lacks phagocytic capability. Furthermore, the erythrocyte membrane has a 2dimensional submembrane cytoskeleton which precludes endocytosis. Therefore, the impetus for the formation of the parasitophorous vacuole must come from the parasite.

Erythrocyte membrane proteins are redistributed at the time of junction formation so that the contact area is free of erythrocyte membrane proteins. A merozoite serine protease which cleaves erythrocyte band 3 has been described.[67]. Because of the pivotal role band 3 plays in the homeostasis of the submembrane skeleton, its degradation could result in a localized disruption of the cytoskeleton. An incipient parasitophorous vacular membrane (PVM) forms in the junction area. This membrane invagination is likely derived from both the host membrane and parasite component and expands as the parasite enters the erythrocyte. Connection between the rhoptries and nascent PVM are sometimes observed. In addition, the contents of the rhoptries are often lamellar (i.e. multilayered) membrane and some rhoptry proteins are localized to the PVM following invasion, suggesting that the rhoptries function in PVM formation.[68]

Ookinetes lack rhoptries and do not form a parasitophorous vacuole within the mosquito midgut epithelial cells. The ookinetes rapidly pass through the epithelial cells and cause extensive damage as they head toward the basal lamina.[69,70] Similarly, sporozoites can enter and exit hepatocytes without undergoing exoerythrocytic schizogony. Those parasites which do not undergo schzogony are free in the host cytoplasm, whereas those undergoing schizogony are enclosed within a PVM.[71] These observations suggest that the PVM is needed for intracellular development and is not necessary for the process of host cell invasion. As the incipient parasitophorous vacuole is being formed, the junction between the parasite and host becomes ring-like and the parasite appear to move through this annulus as it enters the expanding parasitophotous vacuole.

Apicomplexan parasite activity invade host cell and entry is not due to uptake or phagocytosis by the host cell. In addition, the zoites are often motile forms that crawl along the substratum by a type of motility referred to as 'gliding motility'. Gliding motility, like invasion, also involves the release of micronemal adhesions at the posterior end of the zoite. One difference between gliding motility and invasion is that the 
micronemes must be continuously released as the organism is moving. Thus gliding motility does not involve this relatively small moving junction, but a continuous formation of new junctions between the zoite and the substratum. In addition, the adhesins are cleaved from the surface of the zoite as the adhesion reach the posterior of the zotie and a trail of the adhesive molecules are left behind the moving zoite on the substratum. However, the mechanism of motility and invasion are quite similar and thus, during invasion the parasite literally crawls into the host cell through the moving junction. In addition, some apicomplexans use this type of motility to escape from cells and can traverse biological barriers by entering and exiting cells. Cytochalasins inhibit merozoite entry, but not attachment. This inhibition suggests that the force required for parasite invasion is based upon actin-myosin cytoskeletal elements. The ability of myosin to generate force is well characterized (eg.. muscle contraction). A myosin unique to the Apicomplexa has been identified and localized to the inner membrane complex.[72] This myosin is part of a motor complex which is linked to the adhesins. Members of the TRAP family and other adhesins have a conserved cytoplasmic domain. This cytoplamic domain is linked to short actin filaments via aldolase. The actin filaments and myosin are oriented in the space between the inner membrane complex and plasma membrane so that the myosin propels the actin filament toward the posterior of the zoite. The myosin is anchored into the inner membrane complex and does not move. Therefore, the transmembrane adhesins are pulled through the fluid lipid bilayer of the plasma membrane due to their association with the actin filament. Thus the complex of adhesins and actin filaments is transported towards the posterior of the cell. Since the adhesins are either complexes with receptor on the host cell or bound to the substratum, the net result is a forward motion of the zoite. When the adhesins reach the posterior end of the parasite they are proteolyitcally cleaved and shed from the zoite surface.

\section{Summary}

Merozoite invasion is a complex and ordered process. A tentative model of merozoite invasion includes:

1. Initial merozotie binding involves reversible interactions between merozotie surface proteins and the host erythrocyte. The exact roles of MSPI and other merozoite surface proteins are not known.

2. Reorientation by an unknown mechanism result in the apical end of the merozotie being juxtaposed to the erythrocyte membrane.

3. Discharge of the micronemes is coincident with the formation of a tight junction between the host and parasite. The tight junction is mediated by receptor-ligand interactions between erythrocyte surface proteins and integral parasite membrane proteins exposed by microneme discharge.

4. localized clearing of the erythrocyte submembrane cytoskeleton and formation of the incipient parasitophorous vacuole. PVM formation is correlated with the discharge of the rhoptries.

5. movement of the merozoite through the ring-shaped tight junction formed by the receptor/ligand complex. The force is generated by myosin motor associated with the trans-membrane parasite ligands moving along actin filaments within the parasite.

6. Closure of the PVM and erythrocyte membrane 
Many proteins that are involved in the invasion process have been identified. However, much still remains to be learned about the cellular and molecular biology of merozoite invasion.[73,74]

\section{Host erythrocyte modification}

Once inside of the erythrocyte, the parasite undergoes a trophic phase followed by replicative phase. During this intra-erythrocytic period, the parasite modifies the host to make it a more suitable habitat. For example, the erythrocyte membrane becomes more permeable to small molecular weight metabolites, presumably reflecting the needs of an actively growing parasite.

Another modification of the host cell concerns cytoadherence of P. falciparum- infected erythrocyte to endothelial cells and the resulting sequestration of the mature parasite in capillaries and post-capillary veinules. This sequestration likely leads to microcirculatory alteration and metabolic dysfunctions which could be responsible for many of the manifestation of severe falciparum malaria. The cytoadherence to endothelial cells confers at least two advantages for the parasite: 1) a microaerophilic environment which is better suited for parasite metabolism, and 2) avoidance of the spleen and subsequent destruction.

\section{Knobs and cytoadherence}

A major structural alteration of the host erythrocyte are electron-dense protrusion, or 'knobs', on the erythrocyte membrane of P. falciparum cells. The knobs are induced by the parasite and several parasite proteins are associated with the knobs.[75] Two proteins which might participate in knobs formation are the knob-associated histidine rich protein (KAHRP) and erythrocyte membrane protein-2 (PfEMP2), also called MESA. Neither KAHEP nor PfEMP2 are exposed on the outer surface of the erythrocyte, but are localized to the cytoplasmic face of the host membrane. Their exact roles in knob formation are not known, but may involve reorganizing the submembrane cytoskeleton. The knobs are believed to play a role in the sequestration of infected erythrocytes since they are points of contact between the infected erythrocyte and vascular endothelial cells and parasite species which express knobs exhibit the highest levels of sequestration. In addition, disruption of the KAHRP results in loss of knobs and ability to cytoadhere under flow conditions.[76] A polmerophic protein, called PfEMPI, has also been localized to the knobs and is exposed on the host erythrocyte surface. The translocation of PfEMPI to the erythrocyte surface depends in part on another erythrocyte membrane associated protein called PfEMP3.[77] PfEMPI probably functions as a ligand which binds to receptors on host endothelial cells. Other proposed cytoadherence ligand include a modified band-3, called pfalhesin.[78] Seqestrin, rifins and clag9.[79]

PfEMI is a member of the var gene family.[80] The 40-50 var gene exhibit a high degree of variability, but have a similar overall structure. PfEMPI) has a large intracellular N-terminal domain, a transmembrane region and a C- terminal intracellular domain. The C-terminal region is conserved between members of the var family and is believed to anchor PfEMPI to the erythrocyte submembrane cytoskeleton. In particular, this acidic C- terminal domain may interact with the basic KAHRP of the knob[81] as well as spectrin and actin.[82] 
The extracellular domain is characterized by 1-5 copies of Duffy-binding (DBL) domains. These DBL domains are similar to the receptor-binding region of the ligand involved in merozoite invasion (discussed above). The DBL domain exhibit a conserved spacing of cystrine and hydrophobic residues, but otherwise show little homology analysis indicates that there are five distinct classes (designated as $\alpha, \beta, \gamma, \delta \ldots \ldots \ldots . .$. and........) of DBL domains. [83] The first DBL is always the same type (designated $\alpha$ ) and this is followed by a cysteine- rich interdomain region (CIDR). A variable number of DBL in various orders make up the rest of the extracellular domain of PfEMP-I.

\section{Possible Receptors Identified By in Vitro Binding Assays}

- $\quad$ CD36

- Ig Superfamily

- ICAMI

- VCAMI

- PECAMI

- Chondroitin sulfate A

- Heparin sulfate

- Hyaluronic acid

- $\quad$ E- selectin

- Thrombospondin

- Resetting Ligands

- CR-I

- $\quad$ Blood group A Ag

- Glycosaminoglycan

Several possible endothelial receptors have been identified by testing the ability of infected erythrocytes to bind in static adherence assays.[84] One of the best characterized among these is CD36, an $88 \mathrm{kDa}$ intergral membrane protein found on monocytes, platelets and endothelial cells infected erythrocytes from most parasite isolates bind to CD36 and the binding domain has been mapped to the CIDR of PfEMPI. However, CD36 has not been detected on endothelial cells of the cerebral blood vessels and parasites from clinical isolates tend to adhere to both CD36 and intracellular adhesion molecule - 1 (ICAMI). ICAMI is a member of the immunoglobulin superfamily and functions in cell-cell adhesion. In addition, sequestration of infected erythrocytes and ICAMI expression has been co-localized in the brain.[85]

Chondroitin sulfate A (CSA) has been implicated in the cytoadhernce within the placenta and may contribute to the adverse effects of $P$. falciparum during pregnancy. The role of some the other potential receptors is not clear. For example, adherence to thrombospondin exhibits a low affinity and cannot support binding under flow condition. Binding to VCAMI, PECAMI and E- selectin appear to be rare and questions about their constrictive expression on endothelial cells have been raised. However, cytoadherence could involve multiple receptor/ligand interactions.

Resetting is another adhesive phenomenon exhibited by P. falciparum-infected erythrocytes. Infected erythrocytes from some parasite isolates will bind multiple uninfected erythrocytes and PfEMPI appears to have a role in at least some resetting. Possible receptors include complement receptor-I (CRI), blood group A antigen, or glycosaminoglycan moieties on an unidentified proteoglycan. 
The different types of DBL domains and CIDR (discussed above) bind to different endothelial cell receptors[ 86,87 ].For example, DBL, which comprises the first domain, bind to many of the receptors associated with resetting. The binding of the CIDR to CD36 may account for the abundance of this particular binding phenotype among parasite isolates. [88]

\section{Antigenic variation}

The encoding of the cytoadherence ligand by a highly polymorphic gene family presents a paradox in that receptor/ligand interactions are generally considered highly specific. Interestingly, selection for different cytoadherent phenotypes result in a concomitant change in the surface antigenic type.[89] Similarly, examination of clonal parasite lines revealed that change in the surface antigenic type correlated with difference in binding to CD36 and ICAMI. For example, the parental line (A4) adhered equally well to CD36 and ICAMI, whereas one of the A4-derived clones (C28) exhibited a marked preference for CD36.[90] Binding to ICAMI was then reselected by panning the infected erythrocytes on ICAMI. All three parasite clones (A4, C28, C28-I) exhibited distinct antigenic types as demonstrated by agglutination with hyper-immune sera.

The expression of a particular PfEMPI will result in a parasite with a distinct cytoadherent phenotype and this may also affect pathogenesis and disease outcome. For example, binding to ICAM-I is usually implicated in cerebral pathology. Therefore, parasites expressing a PfEMPI which binds to ICAMI may be more likely to cause cerebral malaria. In fact, higher levels of transcription of particular var genes are found in cases of severe malaria as compared to uncomplicated malaria.[91] Similarly, a higher proportion of isolates which bind to CSA are obtained from the placenta as compared to the peripheral circulation of either pregnant women or children.[92] Furthermore, placenta malaria is frequently associated with higher levels of transcription of a particular var gene which binds CSA .[93] This phenomenon is not restricted to the placenta in that, there is a dominant expression of particular var genes in the various tissue.[94] This tissue specific expression of particular var genes implies that different tissues are selecting out different parasite populations based on the particular PfEMPI being expressed on the surface of the infected erythrocyte. To CSA, CD36, or ICAM-I. infected erythrocyte were collected from the placenta, peripheral circulation of the mother, or peripheral circulation of the child. (designated as group 1-6) expressed in different tissues (brain, lung, heart and spleen) from 3 different patients. PM30 died of severe malaria anemia. PM32 was diagnosed with both cerebral malaria and severe anemia. PM55 was diagnosed with only cerebral malaria.

Although sequestration offers many advantages to the parasite, the expression of antigens on the surface of the infected erythrocyte provides a target for the host immune system. The parasite counters the host immune responses by expressing antigenically distinct PfEMPI molecules on the erythrocyte surface. This allows the parasite to avoid clearance by the host immune system, but yet maintain the cetoadherent phenotype. This antigenic switching may occur as frequently as $2 \%$ per generation in the absence of immune pressure. [55] The molecular mechanism of antigenic switching is not known. Experimental evidence indicates that the mechanism is not associated with duplication into specific expression-linked sites as found in African trypanosomes. Only a single var gene is expressed at a time (i.e... allelic exclusion). The non-expressed genes are kept silent by protein which bind to the promoter region. A gene can become activated by repositioning to a particular location in the nucleus 
and is associated with chromatin modification. This expression spot can only accommodate a single active gene promoter. Thus the var promoter is sufficient for both the silencing and the monoclinic transcription of a PfEMPI allele. [ $\left.{ }^{96}\right]$

\section{Summary}

- The malaria parasite modifies the erythrocyte by exporting proteins into the host cell.

- One such modification is the expression of PfEMPI on the erythrocyte surface which functions as cytoadherent ligand.

- The binding of this ligand to receptors on host endothelial cells promotes sequestration and allows the infected erythrocyte to avoid the spleen.

- Numerous PfEMPI genes (i.e... the var gene family) provide the parasite with a means to vary the antigen expressed on the erythrocyte surface.

- $\quad$ This antigenic variation also correlates with different cytoadherent phenotypes.

\section{References}

[1] Chavatte J.M., Chiron F., Landau I. (March 2007). "Probable speciations by "host-vector ' fidelity"': 14 species of Plasmodium from magpies" (in French). Parasite 14 (1): 21-37.

[2] Gueriard P., Tavares J., Thiberge S.,et al. (October 2010). “Development of the malaria parasite in the skill if the mammalian host". Proc. Natl. acad. Sci. U.S.A 107 (43): 18640 - 5

[3] Manson-Bahr PEC, Bell DR, eds. (1987). Manson's Tropical Diseases London: Bailliere Tindall, ISBN 0-7020-1187-8

[4] Cogswell FB (January 1992). "The hypnozoite and relapse in primate malaria." Clinical microbiology reviews 5 (1): 26-35.

[5] Krotoski WA., Collin WE, Bray RS, et al. (November 1982). "Demonstration of hypnozoites in sporozites in sporozoite-transmitted plasmodium vivax infection." The American journal of tropical medicine and hygiene 31 (6): 1291-3.

[6] Greenwood BM, Fidock Da, Kyle DE, et al (April 2008). "Malaria: progress, perils, and prospects for eradication". J. Clin. Onvest. 118 (4): 1266-76.

[7] Tanomsing N, Imwong M, Pukittaykamee S. et al. (October 2007). "Genetic analysis of the dihydrofolate reductase-thymidylate synthase gene from geographically diverse isolates of plasmodium malaria". Antimcrob, Agents Chemother. 51 (10): 3523-30.

[8] Sturm, A; Amino, R; Van De Sand, C; Regen, T; Retzlaff, S; Rennenberg, A; Krueger, A; Pollok, JM; et al. (2006). "Manipulation of host hepatocytes by the malaria parasite for delivery into liver sinusoids". 313 (5791): 1287-1290.

[9] Baer, K; Klotz, C; Kappe, SH; Schnieder, T; Frevert, U; et al. (2007). "Release of hepatic plasmodium yolii merozoite into the pulmonary microvasculature". Plos pathogen 3 (11): e171. doi:10.1371/journal. Ppat. 0030171

[10] Leitgeb AM, Blomqvist K, Cho-Ngwa F, Samje M, Nde P, Titanji V, Wahlgren M. (2011). "Low Anticoagulant heparin disrupts Plasmodium falciparum rosettes in fresh clinical isolates". Am J Trop Med Hyg 84 (3): 390-396.

[11] Tamez PA, Liu H, Fernandez-Pol S, Haldar K, Wickrema A (October 2009). “Stagespecific susceptibility of human erythrocytes to plasmodium falicparum malaria infection". Blood 114 (17): 3652-5.

[12] Muehlenbachs A, Mutabingwa T.K., Fried M., Duffy P.E. (2007) An unusual presentation of placental malaria: A single persisting nidus of sequestered parasites. Hum. Pathol. 38 (3): 520-523, 
[13] Robert V, Boudin C (2003). "Biology of man-mosquito plasmodium transmission". Bull Soc Pathol Exot. 96 (1): 6-20.

[14] Kitchen SF, Putnam P (1942). "Observation on the mechanism of the parasite cycle in falciparum malaria". Am JTrop Med. 22: 381-386

[15] Smalley MEm Sinden RE (1977). Plasmodium falciparum gametocytes: their longevity and infectivity". Parastology 74 (1): 1-8.

[16] Field JW, Shute PG (1956) A morphological study of the erythrocytic parsites. Kuala Lumpur: government Press. The microscopic diagnostic of human malaria; p. 142

[17] Al-Olayana EM, Williamsb GT, Hurd H (2002. “Apoptosis in the malaria protozoan, plasmodium berhei: a possible mechanism for limiting intensity of infection in the mosquito". Inter. J. parasitol 32 (9): 1133-1143.

[18] Hurd H, Grant KM, Arambage Sc (2206). “Apoptosis-like death as a feature of malaria infection in mosquito". Parasitology 132 Suppl: S33-47.

[19] Sologub L, Kuehn A, Kern S, Przyborski J, Schillig R, Pradel G (2011) Malaria proteases mediate inside-out egress of gametocytes from red blood cell following parasite transmission to the mosquito. Cell microbiol. 1462-5822.

[20] Hopwood JA, Ahmed AM, Polwart A Williams GT, Hurd H. (2001). "malaria-induced apoptosis on mosquito ovaries: a mechanism to control vector egg production". J. Exp. Biol. 204 (Pt 16): 2773-2780.

[21] Greenwood, T.; et al. (2008). "Febrile plasmodium falciparum malaria four years after exposure in a man with sickle cell disease". Clin Infect. Dis. 47 (4): e39-e41.

[22] Szmitko, P. E.; Kohn, M L.; Simor, A. E. (2008). "Plasmodium falciparum malaria occurring eight years after leaving an endemic area". Diagn. Microbi Infect. Dis. 61 (1): 105-107.

[23] Theunissen, C.; Mutabingwa, TK; Fried, M; Duffy, PE; Van-Esbroeck, M; Van-Gomple, A, Van-Denende, j. (2009). "Falciparum malaria in patient 9 years after leaving malaria-endemic area". Emerg Infect Dis. 15 (1): 115-116

[24] Poilane I, Jeantils V, Carbillon L (October 2009). “[Pregnancy associated plasmodium falciparum malaria discovered fortuitously: report of two cases]" (in French). Gynecol Obstet Fertil 37 (10): 824-6.

[25] Zenz W, Trop M, Kollaritsch H, Reinthaler (2000) Congenital malaria due to plasmodium falciparum and plasmodium malariae. Wien Klin Wochenschr. 112 (10): 459-461.

[26] Romand S, Bouree P, Gelez J, Bader-Meunier B, Bisaro F, Dommergues Jp (1994) Congenital malaria. A case observed in twins born to an asymptomatic mother Presse Med 23 (17): 797-800

[27] Witkowski B, Lelievre J, Barragan MJ, et al. (May 2010). "Increased tolerance to artemisinin in plasmodium falciparum is mediated by a quiescence mechanism". Antimcrob. Agents. Chemother. 54 (5): 1872-7

[28] Thapar M.M., Gil J.P., Bjorkman A. (2005). In vitro recrudescence of plasmodium falciparum parasite were suppressed to dormant state both by atovaquone alone and in combination with proguanil. Trans R Soc Trop Med Hyg 99 (1): 62-70,

[29] Landau I, Cahbaud AG Mora-Silvera E. et al. (December 1999). "Survival of rodent malaria merozoite in the lymphatic network: potential role in chronicity of the infection." Parasite (Paris, France) 6 (4): 311-22.

[30] Gautret P. (2000). “The Landau and Chabaud's phenomenon". Parasite 7 (1): 57-58.

[31] Moore RB, Obornik M, Janouskovec J, et al. (February 2008). "A photosynthetic alveolate closely related to apicomplexan parasites". Nature 451 (7181): 959-963. 
[32] Moore RB, Obornik M, Janouskovec J, et al. (February 2008). “A photosynthetic alveolate closely related to apicomplexan parasites". Nature 451 (7181): 959-63.

[33] Yotoko, KSC; C Elisei (2006-11). "Malaria parasites (Apicomplexa, Haematozoea) and their relationship with their hosts: is there an evolutionary cost for the specialization?". Journal of Zoological Systematics and Evolutionary Research 44 (4): 265-73

[34] Perkins SL, Schall JJ (October 2002). “A molecular phylogeny of malarial parsites recovered from cytochrome b gene sequences" J. parasitol. 88 (5): 972-8

[35] Yotoko, K.S.C; C Elisei (2006). "Malaria parasites (Apicomplexa, Haematozoea) and their relationship with their hosts: is there an evolutionary cost for the specialization?". Zoo. Syst. Evol. Res. 44 (4): 265

[36] Seethamchai S, Putapornitip C, Malaivijitnond S, Cui L, Jonwutiwes S. (2008). "Malaria and Hepatocystis species in wild macaques, southern Thailand". Am J. Trop Med Hyg 78 (4): 646-653.

[37] Leclerc M.C., Hugot J.P., Durand F. (2004). “Evolutionary relationships between 15 plasmodium species from new and old world primates (including humans): an $18 \mathrm{~S}$ rDNA cladistic analysis". Parasitology 129 (6): 677-684

[38] Mitsui H, Arisue N, Sakihama N. et al. (January 2010). "Phylogeny of Asian primate malaria parasites inferred from apicoplast genome-encoded genes with special emphasis on the positions of Plasmodium vivax and P. fragile". Gene 450 (1-2): 32-8.

[39] Nishimoto Y, Arisue N, Kawai S, Escalante AA, Horii T, Tanabe K, Hashimoto T. (2008).“Evolution and phylogeny of the heterogeneous cytosolic SSU rRNA genes in the genes Plasmodium". Mol Phylogenet Evol. 47 (1): 45-53.

[40] Sawai H, Otani H, Arisue N, Palacpac N, de Oliveira Martins L, Pathirana S, Handunnetti S, Kawai S, Kishino H, et al. (2010). “Lineage-specific positive selection at the merozoite surface protein I (mspl) locus of plasmodium vivax and related simian malaria parsites". Evol Biol. 10 (1): 52.

[41] Escalante AA Cornejo OE, Freeland DE, Poe AC, Durrego E, Collins We, Lal AA. (2005). "Amonkey's tale: the origin of plasmodium vivax as a human malaria parasite". Proc Natl Acad Sci USA 102 (6): 1980-5.

[42] Kishore SP, Perkins Sl, Templeton TJ, Deitsh KW. (June 2009). “An unusual recent expansion of the C-terminal domain of RNA polymerase II in primate malaria parasites features a motif otherwise found only in mammalian polymerases". $J$. Mol. Evol. 68 (6): 706-14.

[43] Prugnolle F, Durand P, Neel C, Ollomo B, Ayala,FJ, Arnathau C, Etienne L, MpoudiNgole E, Nkoghe D et al (2010). "African great apes are natural hosts of multiple related malaria species, including plasmodium falciparum". Proc. Natl. Acad. Sci. USA 107 (4): 1458-1463.

[44] Duval L, Fourment M, Nerrienet E, et al (June 2010). “ African apes as reservoirs of plasmodium falciparum and the origin and diversification of the Laverania subgenus". Proc. Natl. Acad. Sci. USA 107 (23): 10561-6.

[45] Liu W, Li Y, Learn GH, Rudicell RS, Robertson JD, Keele, BF, Ndjango JB, Sanz CM, Morgan DB et al (2010). "Origin of the human malaria plasmodium falciparum in gorillas". Nature 467 (7314).

[46] Rich SM, Leendertz FH, Xu G, et al (September 2009). “The origin of malignant malaria". Proc. Natl. Acad. Sci. U.S.A 106 (35): 14902-7

[47] Ricklefs RE, Outlaw DC, (2010). “A molecular clock for malaria parasites". Science 329 (5988): 226-229. 
[48] Sutherland CJ, Tanomsing N, Nolder D, Oguike M, Jennison C, Pukrirrayakamee S, Dolecek C, Hien TT, do Rosario VE, Arez AP, Pinto J Michon P, Escalante AA, Nosten F, Burke M, Lee R, Blaze M, Otto TD, Barnwell JW, Pain A, Williams J, White NJ, Day NP, Snounou G, Lockhart PJ, Chiodini Pl, Imwong M, Polley SD, (2010). "Two non-recombining sympatric forms of the human malaria parsite Iplasmodium ovale occur globally". J Infect Dis 201 (10): 1544-50.

[49] dos Santos LC, Curotto SM, de Moraes W, et al. (July 2009). "Detection of plasmodium sp, in capybara". Vet parsitol 163 (1-2): 248-51.

[50] Corradetti A,; Garnham P, C. C; Laird M. (1963). "New classification of the avian malaria parasites". Parassitologia 5: 1-4 ISSN 0048-2951.

[51] Martinesn ES, Waite JL, Schall JJ (April 2007). "Morphologically defined subgenera of plasmodium from avian hosts: test of monophyly by phylogenetic analysis of two mitochondrial genes". Parasitology 234 (Pt4): 483-90

[52] Telford, S. (1998). "A contribution to the systematic of the reptilian malaria parasites, family Plasmodium (Apicomplexa: Haemosporing". Bulletin of the Flroida State Museum Biological Sciences 34 (2): 65-96.

[53] Gratzer WB, Dluzewski AR (1993) The red blood cell and malaria parasite invasion. Semin Hematol 30, 232-247

[54] Holder AA, Blackman MJ, Borre M, Burghaus PA, Chappel JA, Keen JK, Ling IT, Ogun SA, Owen CA, Sinha KA (1994) Malaria parasite and erythrocyte invasion. biochem Soc Trans 22, 291-295.

[55] Goel VK, Li X, Liu SC, Chishti AH, Oh SS (2003) Band 3 is a host receptor binding merozite surface protein I during Plasmodium falciparum invasion of erythrocytes. Proc Natl Acad Sci 100, 5164-5169.

[56] Sinnis P, Sim BKL (1997) Cell invasion by the vertebrate stages of plasmodium. $\mathrm{Tr}$ Microbiol 5, 52-58.

[57] Cooper JA (1993) Merozoite surface antgen-1 of Plasmodium. Parasitol Today 9, 50-54.

[58] Mitchell GH, Thomas AW, Margos G, Dluzewski AR, Bannister LH (2004) Apical membrane antigen 1, a major malaria vaccine candidates, mediated the close attachment of invasive merzoites to host red blood cells. Infect. Immune. 72, 154-158.

[59] Carruthers VB, Sibley LD (1997) Sequential protein secretion from three distinct organelles of Toxoplasma gondii accompanies invasion of human fibroblasts. Eur. J Cell Biol 73, 114-123

[60] Carruthers VB, Sibley LD (1999) Mobilization of intracellular calcium stimulates microneme discharge in Toxoplasma gondii. Mol Microbilo 31, 421-428

[61] Culvenor JG, Day KP, Anders RF (1991) P. falciparum ring-infected erythrocyte surface antigen is released from merozoite dense granules after erythrocyte invasion. Infect immune 59, 1183-1187

[62] Blackman MJ, FuJioka H, Stafford WE, Sajid M, Clough B, Fleck SL, Aikawa m< Grainger M, Hackett F (1998) A subtilism-like protein in secretory organelles of plasmodium falciparum merozites. J Biol Chem 273, 23398-23409

[63] Barale JC, Blisnick T, Fujioka H, Alzari PM, Aikawa M, Braun,-Breton C, Langsley G (1999) Plasmodium falciparum subtilism-like protease 2, a merozite candidate for the merozoite surface pertein 1-42 maturase Proc Natl Acad Sci U S A 96, 6445-6450.

[64] Reed Mb, Caruana SR, Batchelor AH, Thompson JK, Carbb BS, Cowman AF (2000) Targeted disruption of an erythrocyte binding antigen in plasmodium falciparum is associated with a switch toward a sialic acid-independent pathway if invasion. proc Natl Acad Sci USA 97, 7509-7514. 
[65] Adams JH, Sim BKL, Dolan SA, Fang XD, Kaslow DC, Miller LH (1992). A family of reythrocyte binding protein of malaria parasites. Proc Natl Acad USA 89, 7085-7089

[66] Tomley FM, Soldati DS (2001) Mix and match modules: structure and function of microneme proteins in apicomplexan parasites, trends Parasitol. 17, 81-88

[67] Braun-Breton C, Pereira da Silva LH (1993) Malaria proteases and red blood cell invasion. parasitol Today 9, 92-96.

[68] Sam-Yellowe TY (1996) Rhoptry organelles of the apicompexa: their role in host cell invasion and intercellular survival. Today 12, 308-316

[69] Han YS,Thompson J, Kafatos FC, Barillas-Mury C (2000) Molecular interactions between Anopheles stephensi midgut cells and plasmodium berghei: the time bomb theory of ookinete invasion of mosquitoes. EMBO J 19, 6030-6040

[70] Waller KL, Cooke BM, Nunomura W, Mohandas N, Coppel RL (1999) Mapping the binding domains involved in the interaction between the Plasmodium falciparum konb-associated histidine-rich protein (KAHRP) and the crtoadherence ligand $P$. falciparum erythrocyte membrane protein-I (PfEMPI). J Biol Chem 274, 23808-23813

[71] Mota MM, Pradel G, Vanderberg GP, Hafalla JCR, Frevert U, Nussenzweig RS, Nussenzweig V, Rofriguez A (2001) Migration of Plasmodium sporozites through cells before infection. Science 291, 141-144

[72] Kappe SHI, Buscaglia CA Bergman LW, Coppens I, Nussenzweig V (2004) Apicomplexan gliding motility and host cell invasion: overhauling the motor model. Trends in Parasitology 20,13-16.

[73] Iyer J, AC Gruner, L Renia, G Snounou and PR Preiser (2007) invasion of host cells by malaria parasite: a tale of two protein families. Molecular Microbiology 65:231-249.

[74] Baum J, Gilberger TW, Frischknecht F, Meissner M (2008). Host-cell invasion by malaria parasite: insights from Plasmodium and Toxoplasma. Trends in parasitology 24, 557563.

[75] Deitsch KW, del Pinal A, Wellems TE (1996) membrane modifications in erythrocytes parasitized by plasmodium falciprum. Mol. Biochem Parasitol 76, 1-10

[76] Crabb BS, Cooke BM, Reeder JC, Waller RF, Caruana SR, Davern KM, Wickham ME, Brown GV, Coppel RL, Cowman AF (1997) Targeted gene disruption shows that knobs enable malaria-infected red cells to cytoadhere under physiological shear stress. Cell 98, 287-296.

[77] Waterkeyn JG, Wickham ME, Davern KM, Cooke BM Coppel RL, Reeder JC, Culvenor JG, Waller RF, Cowman AF (2000) Targeted mutagenesis of Plasmodium falciparum erythrocyte membrane protein 3 (PfEMP3) disrupts cytoadherence of malariainfected red blood cells EMBO J 19, 2813-2823.

[78] Sherman IW, Eda S, Winograd E (2003) Cytoadherence and sequestration in Plasmodium falciparum: defining the ties that bind, microbes and infection 5, 897-909

[79] Craig A, Schert A (2001) Molecules on the surface of the Plasmodium falciparum infected erythrocyte and their role in malaria pathogenesis and immune evasion. Mol. Biochem. Parasitol. 115, 129-143.

[80] Deitsch KW, del Pinal A, Wellems TE (1999) intra-cluster recombination and var transcription switches in the antigenic variation plasmodium falciparum. Mol Biochem Parasitol 101, 107-116.

[81] Bannister LH, Mitchell GH, Butcher GA Dennis ED, Cohen S (1986). Structure and development of the surface coat of erythrocytic merozites of plasmodium knowlesi. Cell Tissue Res 245, 281-290 
[82] Oh SS, Voigt S, Fisher D, Yi SJ, LeRoy PJ, Derick LH, Liu SC, Chishti Ah (2000) Plamodium falciparum erythrocyte membrane protein 1 is anchored to spectrin-actin junction and knob-associated histidine-rich protein the erythrocyte cytoskeleton. Mol Biochem Parasitol 108, 237-247

[83] Deitsch KW, del Pinal A, Wellems TE (1999) intra-cluster recombination and var transcription switches in the antigenic variation plasmodium falciparum. Mol Biochem Parasitol 101, 107-116.

[84] Beeson JG, Brown GV (2002). Pathogenesis of Plasmodium falciparum malaria: the roles of parasite adhesion and antigenic variation. Cell, Mol. Life Sci. 59,258-271

[85] Turner GDH, Morrison H, Jones M, Davis TEM, Looareesuwan S, Buley ID, Gatter KC, Newbold CI, Pukritaykamee S, Nagachinta B, White HJ, Berendt AR (1994) An immunohistochemical study of the pathology of fatal malaria - evidence for widespread endothelial activation and a potential role for intercellular adhesion molecule-I in cerebral sequestration. Am J Pathol 145, 1057-1069.

[86] Deitsch KW, del Pinal A, Wellems TE (1999) intra-cluster recombination and var transcription switches in the antigenic variation plasmodium falciparum. Mol Biochem Parasitol 101, 107-116.

[87] Craig A, Schert A (2001) Molecules on the surface of the Plasmodium falciparum infected erythrocyte and their role in malaria pathogenesis and immune evasion. Mol. Biochem. Parasitol. 115, 129-143.

[88] Sherman IW, Eda S, Winograd E (2003) Cytoadherence and sequestration in Plasmodium falciparum: defining the ties that bind, microbes and infection 5, 897-909

[89] Biggs BA, Anders RF, Dillon HE, Davern KM, Martins M, Petersen C, Brown GV (1992) Adherence of infected erythrocytes to venular endothelium selects for antigenic variants of plasmodium falciparum J Immunol 149, 2047-2054.

[90] Roberts DJ, Craig AG, Berendt AR, Pinches R, Nash G, Marsh K, Newbold CI (1992) Rapid switching to multiple antigenic and adhesive phenotpes in malaria. Nature 357, 689-692.

[91] Rottmann M, Lavstsen T, Mugasa JP, Kaestli M, Jensen ATR, Muller D, Theander T, Beck HP (2006) Differential expression of var gene groups is associated with morbidity caused by Plasmodium falciparum infection in Tanzanian children. Infect. Immune. 74, 3904-3911

[92] Beeson JG, Brown GV, Molneux Me, Mhango C, Dzinjalalala F, Rogerson SJ (1999) Plasmodium falciparum isolates from infected pregnant woman and children are associated with distinct adhesive and antigenic properties. J Infect Dis 180,464-472.

[93] Duffy EF, Caragounis A, Noviyanti R,. Kyriacou HM, Choong EK, Boysen K, Healer J, Rowe JA, Molyneux EM, Brown GV, Rogerson SJ (2006) Transcribed var genes associated with placental malaria in Malawian women. Infect. Immune. 74:487-4883.

[94] Montgomery J, Mphande FA, Berriman M, Pain A, Rogerson SJ, Taylor TE, Molyneux ME, Craig A (2007) Differential var genes expression in the organs of patients dying of falciparum malaria. Molecular Microbiology 65, 959-967.

[95] Scherf A, Hernandez-Rivas R, Buffet P, Bottius E, Benatar C, Pouvelle B, Gysin J, Lanzer M (1998) Antigenic variation in malaria: in situ switching, relaxed and mutually exclusive transcription of var genes during intra-erythrocytic development in Plasmodium falciparum. EMBO J 17, 5418-5426.

[96] Voss TS, Healer J, Marty AJ, Duffy MF, Thompson JK, Beeson JG, Reeder JC, Crabb BS, Cowman AF (2006) A var gene promoter controls allelic exclusion of virulence genes in Plasmodium falciparum Malaria. Nature 439: 1004-1008. 


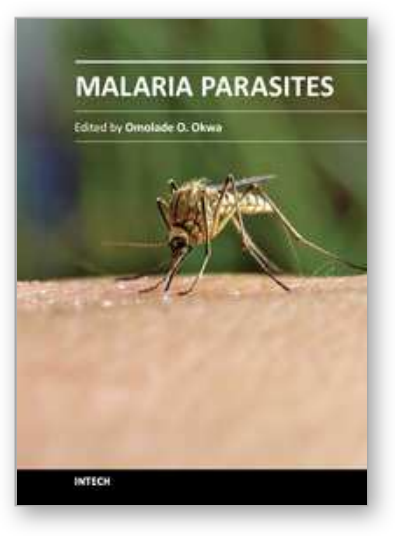

\author{
Malaria Parasites \\ Edited by Dr. Omolade Okwa
}

ISBN 978-953-51-0326-4

Hard cover, 350 pages

Publisher InTech

Published online 30, March, 2012

Published in print edition March, 2012

Malaria is a global disease in the world today but most common in the poorest countries of the world, with $90 \%$ of deaths occurring in sub-Saharan Africa. This book provides information on global efforts made by scientist which cuts across the continents of the world. Concerted efforts such as symbiont based malaria control; new applications in avian malaria studies; development of humanized mice to study P.falciparium (the most virulent species of malaria parasite); and current issues in laboratory diagnosis will support the prompt treatment of malaria. Research is ultimately gaining more grounds in the quest to provide vaccine for the prevention of malaria. The book features research aimed to bring a lasting solution to the malaria problem and what we should be doing now to face malaria, which is definitely useful for health policies in the twenty first century.

\title{
How to reference
}

In order to correctly reference this scholarly work, feel free to copy and paste the following:

John C. Igweh (2012). Biology of Malaria Parasites, Malaria Parasites, Dr. Omolade Okwa (Ed.), ISBN: 978953-51-0326-4, InTech, Available from: http://www.intechopen.com/books/malaria-parasites/biology-ofmalaria-parasites

\section{INTECH}

open science | open minds

\section{InTech Europe}

University Campus STeP Ri Slavka Krautzeka 83/A 51000 Rijeka, Croatia Phone: +385 (51) 770447

Fax: +385 (51) 686166 www.intechopen.com

\section{InTech China}

Unit 405, Office Block, Hotel Equatorial Shanghai No.65, Yan An Road (West), Shanghai, 200040, China 中国上海市延安西路65号上海国际贵都大饭店办公楼 405 单元 Phone: +86-21-62489820

Fax: +86-21-62489821 
(C) 2012 The Author(s). Licensee IntechOpen. This is an open access article distributed under the terms of the Creative Commons Attribution 3.0 License, which permits unrestricted use, distribution, and reproduction in any medium, provided the original work is properly cited. 